\title{
One of the most fascinating stories in biology
}

\author{
Friedrich G. Barth ${ }^{1}$
}

Received: 1 May 2019 / Accepted: 13 May 2019 / Published online: 23 May 2019

c) Springer-Verlag GmbH Germany, part of Springer Nature 2019

\begin{abstract}
Science advances but slowly, with halting steps. But does not therein lie her eternal fascination? And would we not soon tire of her if she were to reveal her ultimate truths too easily?
\end{abstract}

Karl von Frisch, Nobel laureate and founder of the Journal of Comparative Physiology, in "A biologist remembers", 1967.

The partnership between insects and flowers in pollination is among the most fascinating stories in biology. It is a show case of organismic diversity and the working of evolution. Apart from attracting our intrinsic intellectual interest and fascination, it is of prime ecological significance and has an enormous economic impact as well. Research into this partnership, which is an opportunistic business trading food against pollination, is not new at all, but started more than 200 years ago (Sprengel 1793). Accordingly, a large number of cases of fine-tuned adaptedness to this business at all levels of organismic organization have come to light already. This applies to both the flower and the pollinator side, which both are subject to selection pressures working in favor of survival and reproductive success. Considering the staggering diversity of angiosperms and even more so that of insects, the spectrum of specializations is enormous, sometimes just beautiful for us like the colors of an alpine meadow at summertime, sometimes bizarre and hard to believe, like the stories about fraud and robbery. To reach a well-founded understanding of adaptedness and the selective pressures that have shaped it research approaches need to be both reductionist and holistic. What we see today largely is the product of some 100 million years of co-evolution. In this sense, the summer meadow to a considerable extent is to be valued as a reflection of the pollinating insects and vice versa. This is seen in countless intimate dependencies resulting from reciprocal selection.

Friedrich G. Barth

friedrich.g.barth@univie.ac.at

1 Department of Neurobiology, Faculty of Life Sciences, University of Vienna, Althanstr.14, 1090 Vienna, Austria
In this special issue of the Journal of Comparative Physiology A some recent research will be highlighted dealing with such different subjects as evolutionary fundamentals, functional morphology, floral signals, and insect senses and cognition.

To start, Hannes Paulus in his review 'General questions and sexually deceptive orchids' raises some basic questions regarding evolutionary mechanisms, which are crucial for an understanding of the partnership between insects and flowers and the meaning of biodiversity. How can the reciprocal selection generating a particular pollination syndrome be reliably proven? The European orchids of the sexual deceptive genus Ophrys are taken as an example. Their flowers do not offer any food and have developed fascinating ways of manipulating their pollinators. They demonstrate the complexity and refinement in the relationship between flower signals attracting male bees and wasps and the visitors' responses, even making use of the pollinator's ability to memorize and learn an individual flower's characteristics. The flower fools its highly species-specific insect visitor when attracting him by visual, chemical and tactile signals, which he expects to stem from a receptive conspecific female. However, the male insect remembers and avoids a flower already visited, associating it with an unreceptive female. These findings point to the relevance of the striking variability of both the visual and olfactory signals emanating from the flowers. In the end, the flowers' variability supports cross-pollination and helps to avoid self pollination. The pollinator's innate preference is overruled by learning. Clearly, this story also underlines the value and necessity of combining simple observations and manipulative experiments in the field with work in the laboratory, learning from analyses made possible by modern technology.

Such technology has proven crucial with regard to the detailed analyses of many floral scents and the chemosensory capabilities of the corresponding insect visitors.

'Floral scent, nectar and chemoreception' is the headline for the following four contributions. Annemarie Heiduk et al. present another very specialized pollination system where floral scents are a crucial element in the communication between the partners. Ceropegia stenantha 
(Apocynaceae), a climbing plant in Africa, has deceptive pitfall flowers used to trap scavenger flies (Coboldia fuscipes, Scatopsidae). Remarkably, the plant mimics volatiles which are also released by distressed bees and attract the carnivorous flies. In other words, the flowers of this plant mimic a food source of the flies. How clever they are!

The subject of the chapter by Thomas Eltz et al. is less of a criminal story. Its focus is on a group of insects with outstandingly long proboscides and very often a particularly beautiful metallic green or blue color: the orchid bees (Euglossini). Their males collect odoriferous chemicals from various sources including orchids and store them in elaborate hind-leg pouches. While collecting at orchids they pollinate them. Later, they release these substances at mating sites, presumably to communicate with other males or to attract females. Since the exact composition of the stored mixture may contain various information valuable in intraspecific communication, the authors for the first time studied the changes in quantity and composition over time to get a better idea of the perfume's potential behavioral meaning.

Considering that insect pollinators, too, need amino acids for protein synthesis and that different amino acids should be balanced in their diet, Fabian Ruedenauer et al. ask whether bumblebees are able to perceive and distinguish amino acids by chemotactile stimulation of their antennae. Obviously, bumblebees possess amino acid receptors on their antennal tips and are indeed likely to be able to assess the overall amino acid contents of pollen and nectar before ingesting them and thus to select for high quality. They cannot, however, differentiate between the different amino acids they perceive. The possibility remains that receptors on body parts other than the antennae are involved as well or even stimulated post-ingestively only.

In another study based on the fact that nectar is not just sugar water but instead contains a complex mixture of compounds, including amino acids, sometimes aversives and products due to bacterial activity, Elizabeth Nicholls et al. go one step further. Using honeybees they examine the reward properties of artificially mixed "nectars" with pre-ingestive taste and learning assays, asking for functional consequences like foraging decisions of non-sugar components such as amino acids and distasteful substances. When adding pollen (the main source of amino acids for bees and containing many more substances) some undefined compounds of it detracted from the perceived value of the sucrose reward and devaluated it. The data presented add to an increasing body of knowledge of complex effects (like the defense against herbivory and robbery) floral rewards may have by varying their composition.

Our next section comes under the headline 'Vision, floral colors and image statistics'. Among the signals used by flowers to attract pollinators, visual ones play a prominent role in many cases, typically being an attractant noticed from a distance. Adrian Dyer et al. present a study on innate color preference using an Australian stingless bee, the sugarbag bee Tetragonula carbonaria, which pollinates a food deceptive orchid endemic to south-eastern Australia with white and pink flower morphs. These are believed to mimic food rewarding flowers found in the same habitat. The basic question is how color signals evolve and how the orchids exploit the visual capabilities and behavior of the pollinator. Explicitly, the authors also use wild flowers in their natural setting instead of only artificial stimuli pointing to the persisting value and necessity of keen observations in the field. Their findings with food deceptive Caladenia carnea show an interesting difference to the findings described in the contribution of Hannes Paulus for the sexually deceptive Ophrys heldreichii species. There is no habituation effect toward unrewarding Caladenia flowers. However, when switching from the non-preferred pink to the innately preferred white color the number of landings significantly increased.

Casper van der Kooi and associates are interested in how light is manipulated by photonic structures responsible for floral coloration. They reveal the intriguing functional morphology and related physical basis of the colors of poppies. These are remarkably bright, despite the thinness (they are only three cell layers thick) and floppiness of their petals. Their reflectance value for long wavelengths nevertheless measures some $35 \%$. Dense pigmentation and strongly scattering structures including air cavities and curvy cell walls are found to mainly explain the phenomenon.

Finally, Olga Dyakova et al. remind us that natural scenes may not be so overwhelmingly rich in information as not to be possibly handled adequately by any visual system. There are ways to simplify the input. But what are the coding adaptations and what is their behavioral relevance? Natural scenes indeed are not as random as we are inclined to think but are constrained in space and time, which can be shown by the image statistics of corresponding photographs. Importantly, it first has to be defined what "natural" means for a particular animal's scene and specific behavior. The authors focus on a hoverfly (Episyrphus balteatus, Syrphidae), which is a commercially important pollinator and aphid controller. They examine scenes typically associated with hovering and free flight, respectively. On a quantitative basis, they point to the differences and the importance of considering the relevant behavior when defining what is "natural".

This special issue in a modest way also gives an impression of the enormous diversity of the insect pollinators. The subjects of research are not only the honeybee (likely to be among the three most intensively studied of all insect species) but also bumblebees, euglossine bees, stingless bees, hoverflies, carpenter bees and hawkmoths, the latter two being the focus of the two chapters under the simple headline 'Carpenter bees and hawkmoths'. 
Hema Somanathan et al. review the foraging strategies and related sensory, physiological and cognitive adaptations known in the impressively large carpenter bees (Xylocopinae). These are common and extremely generalist flower visitors in the tropics and subtropics worldwide and clearly have not received the attention yet they should have had. Among other things, we learn that they are traplining and long distance foragers, implying the likelihood of well-developed navigational skills. Their remarkable thermoregulatory capabilities are suggested to be important for foraging at dim light conditions, which also goes along with visual adaptations of their apposition eyes in the species flying after sunset. Eye structure and anatomy of three sympatric Xylocopa species with different visual ecology (nocturnal, crepuscular, diurnal) differ significantly.

There are about 1500 species of hawkmoths (Spingidae), well known for the hovering in front of the flower when collecting nectar as shown by many of them. This behavior is both costly regarding energy consumption and demanding regarding the mechanisms of flight control. Approaching the plant with extended proboscis the hawkmoth has to find a sometimes very narrow entrance to the nectar. With few exceptions, most hawkmoths are active in twilight or nocturnal. Anna Lisa Stöckl and Almut Kelber review the rich knowledge available of the senses (visual, chemical, mechanical) and sensory-guided strategies which hawkmoths use when searching for flowers, exploiting a nectar source and maintaining their position while hovering. This implies a review of the signals and cues associated with the plants and attracting their insect visitors. A general conclusion of the authors maintains that research on hawkmoth sensory ecology is now well prepared to go to the field and to emphasize real life stimuli instead of the reductionist stimuli largely applied under laboratory conditions. This certainly will open up exciting new avenues for future research.

A review paper by Michael Hrncir et al. elaborates on the impact and challenges of 'Living in an extreme habitat' for a eusocial bee. Melipona subnitida belongs to the stingless bees (Meliponini), which amount to some 500 species and are the most important pollinators in the tropics and subtropics. M. subnitida lives in the tropical dry forest (Caatinga) in the north-east of Brazil. It is usually rather hot there and rain may be missing for several months or much longer, limiting the availability of floral food to only short and unpredictable periods. Apart from heat resistance, how do these highly eusocial bees manage to maintain perennial colonies in such a hot and semi-arid environment? One of the main tricks is a remarkably drastic reduction of brood production and adult activity during the dry period and the ability to quickly up-regulate reproductive activity and to reactivate the colony when the rain again permits to collect and store pollen and nectar.
Learning and memory are crucial determinants of an insect's success to find and exploit floral resources efficiently. Accordingly, they have been the subject of a wealth of research and indeed contributed essentially to raise and answer questions of general importance and relevance in neurobiology and neuroethology. The number of traits used by flowers to attract or repel insects and the attention of biologists has recently increased. Temperature patterns, electric fields and anti-aphrodisiac compounds in nectar are examples that come to mind. Our last section entitled 'Cross-modal transfer and numerosity' deals with two rather advanced neural capabilities.

Michael Harrap et al. address the issue of multimodality. Obviously, the display of plants is multimodal, their flowers presenting several signal types simultaneously. In line with this, bumblebees have been shown to readily recognize and distinguish not only flowers differing in visual patterns, but also in patterns of scent, surface texture, temperature and electrostatic field. Importantly, they can transfer learnt patterns between different stimulus modalities without the necessity to relearn, concomitantly increasing the effectivity of the flower's signals. This is at least true for non-visual scent patterns and similar visual patterns. It does, however, not apply to visual patterns and temperature patterns, indicating that cross-modality learning transfer is not a property universal for all types of patterned signals. The authors discuss this and, among other things, point to differences between the closeness of neural links of stimulus modalities in the brain. Fortunately, research now increasingly attends to questions related to multimodal neural integration and perception.

The final contribution of this Special Issue is dedicated to a phenomenon which has much been debated lately. Are honeybees able to process numbers? Martin Giurfa reviews the ongoing debate. It has been shown that the tiny brain of the honeybee, already known to have impressive cognitive abilities such as category and concept learning, is capable of numerosity judgements, that is to learn a rule like "smaller than" or "larger than" and even to use zero as the low end of their numerical continuum. There are several interesting analogies with the numeric system of vertebrates. Selective pressures which may have led to the numerical competence of honeybees may have been the advantages provided by navigation skills like the ability to estimate landmark numbers on the way to the food, the number of flower arrays and the number of recruits successfully activated. As Martin Giurfa explains, the miniaturization of an insect brain does not go along with a lack of sophistication. However, a lot has still to be learned to understand the neural mechanisms underlying this sophistication and their evolutionary origin.

As is evident from the contributions to this Special Issue, pollination biology is a highly synthetic discipline, bringing together plants and animals, ecology, neuro- and sensory 
biology, neuroethology, behavioral ecology, chemistry, physics and more. It has to ask both proximate questions and ultimate evolutionary questions, which are not always easy to separate from each other. To fully understand all these masterpieces of adaptations, we need both the details and the more holistic view of the organisms embedded in their organic and inorganic worlds. Regarding the dramatic loss of biodiversity which we currently experience and the topic of this Special Issue, we must not forget the fundamental service of insects to ecosystems and the need of both partners to live their specific life in their species-specific environments. Instead of exploiting nature as if it were an endless self-service buffet we should relearn to see us as a part only of a big fragile network, on which we heavily depend. And also: Aren't we poor without the beauty we find in nature left alone and flourishing?

\section{References}

Sprengel Ch K (1793) Das entdeckte Geheimnis der Natur im Bau und in der Befruchtung der Blumen. Friedrich Vieweg dem aeltern, Berlin, 447 pp, 25 copper plates. Reprint 1972: Cramer J, Swann HK (eds) Historiae naturalis classica, XCVII. Verlag J Cramer, 3301 Lehre

von Frisch K (1967) A biologist remembers. Pergamon Press, Oxford

Publisher's Note Springer Nature remains neutral with regard to jurisdictional claims in published maps and institutional affiliations. 